\title{
Advances in Sintering
}

\author{
M. QIAN ${ }^{1,2}$ \\ 1.-Centre for Additive Manufacturing, School of Aerospace, Mechanical and Manufacturing \\ Engineering, RMIT University, Melbourne, VIC, Australia. 2.-e-mail: ma.qian@rmit.edu.au
}

The global powder metallurgy market has continued to benefit from the revival in the automotive industry while emerging applications are expected to further drive demand for powder metallurgy $(\mathrm{PM})$ components in the near future. Sintering is the mainstay of a typical PM process in the production of various types of PM components, whether they are pore-free, nearly pore-free, or porous. At the committee meeting held in March 2015, the TMS Powder Materials Committee decided to choose sintering as the committee's technical emphasis for JOM 2016. Six papers were selected for the JOM March 2016 topic following a thorough review process.

In the first article entitled "Sintering Trajectories: Description on How Density, Surface Area, and Grain Size Change," German discusses the ways how the sintering process has been treated by researchers in the PM community. As pointed out by the author, an alternative approach to the theoretical treatment using a matrix of mathematical relationships is to track the sintering trajectory using relatively simple relationships based on bulk measures, including density, surface area, and grain size. These relationships, although they often largely ignore mechanistic details, are applicable to a wide variety of materials and consolidation conditions, including hot pressing and spark sintering. It is suggested that, over a broad array of metals and ceramics, the sintering trajectories follow a characteristic trajectory, where specific surface area, grain size, and fractional density are interrelated.

The second article, "Sintering in Laser Sintering" by Bourell, offers an informative discussion of the term 'laser sintering' and its accuracy in the broad context of powder metallurgy including additive manufacturing. It seems clear from this contribution that the PM community might consider laser "sintering" to be a historical term and a misnomer.

Ma Qian is the JOM advisor for the Powder Materials Committee of the TMS Materials Processing \& Manufacturing Division, and guest editor for the topic Advances in Sintering in this issue.
Should "laser sintering" be called something else? In the interests of accuracy, this is probably so according to the author.

The third article in this sequence, by Wang et al., deals with the "Fabrication of High Strength and Ductile Stainless Steel Fiber Felts by Sintering." Stainless steel fiber felts are important porous stainless steel products, which have found wide applications for high-volume filtration or separation, sound absorption or noise control, heat transfer, and energy absorption, as well as being used in catalytic converters and surface combustion. This original research article presents a comprehensive experimental study of the fabrication of stainless steel fiber felts by sintering. The critical sintering conditions for the formation of undesired bamboolike grain structures in the fiber ligaments are defined and an innovative sintering process is proposed and demonstrated.

The fourth article, by Liu and co-workers, reports on the development of "Ultrahigh Strength and Ductile Lamellar-Structured Powder Metallurgy Binary Ti-Ta Alloys." Ti-Ta alloys are expected to offer better biocompatibility and corrosion resistance than titanium, especially when the Ta content exceeds about 15 at.\% or 40 wt.\%. The binary Ti20at.\% Ta alloy fabricated by the authors via sintering from elemental $\mathrm{Ti}$ and Ta powders and subsequent hot-swaging and annealing achieved ultimate tensile strength of $1600 \mathrm{MPa}$ and tensile elongation of greater than $25 \%$. The microstructure of the asfabricated Ti-20at.\% Ta alloy is featured by aligned Ta-enriched and Ti-enriched phases, which originated from the insufficient diffusion of Ta during isothermal sintering, an interesting approach adopted by the authors.

In the fifth article in this compilation, Dvilis and co-workers investigate the consolidation by spark plasma sintering (SPS) of multi-phase metal matrix $(\mathrm{Al} / \mathrm{Mg})$ powder composites. The research focuses on modeling and experimental verification of the consolidation process of powder composites consisted of aluminium-magnesium alloy AMg6 (65 wt.\%), B4C 
powder (15 wt.\%), and W nano-powder (20 wt. \%), as well as the optimization of both the composite content and SPS conditions for improved consolidation.

For most metal powders (titanium powder is an exception), the oxide film has an important effect on their sintering behaviors including the selection of sintering temperature. In the sixth and final article in this collection, Gierl-Mayer and co-authors provide an informative review of 'The Role Of Oxygen Transfer In Sintering Of Low Alloy Steel Powder Compacts' and discuss in detail the influence of alloying elements which have higher oxygen affinity than iron. Several important conclusions and recommendations are made at the end of the review.

In summary, this selection of articles highlights a number of different aspects of sintering. To download any of the papers, follow the URL:http://link. springer.com/journal/11837/68/3/page/ 1 to the table of contents page for the March 2016 issue (Vol. 68, No. 3).
- "Sintering Trajectories: Description on How Density, Surface Area, and Grain Size Change," R.M. German

- "Sintering in Laser Sintering," D. Bourell

- "Fabrication of High Strength and Ductile Stainless Steel Fiber Felts by Sintering," J.Z. Wang, H.P. Tang, M. Qian, A.J. Li, J. Ma, Z.G. Xu, C.L. Li, Y. Liu, and Y. Wang

- "Ultrahigh Strength and Ductile LamellarStructured Powder Metallurgy Binary Ti-Ta Alloys,” Y. Liu, S. Xu, X. Wang, K. Li, B. Liu, H. Wu, and H.P. Tang

- "Spark Plasma Sintering of Aluminum-Magnesium-Matrix Composites with Boron Carbide and Tungsten Nanopowder Inclusions: Modeling and Experimentation," E.S. Dvilis, O.L. Khasanov, V.N. Gulbin, M.S. Petyukevich, A.O. Khasanov, and E.A. Olevsky

- "The Role of Oxygen Transfer in Sintering of Low Alloy Steel Powder Compacts: The "Internal Getter" Effect," C. Gierl-Mayer, R. de Oro Calderon, and H. Danninger 